University of Windsor

Scholarship at UWindsor

$12-17-2020$

\title{
Preferential reaching and End-State Comfort: How task demands influence motor planning.
}

\author{
Danielle Salters \\ University of Windsor \\ P. Camila Rios \\ University of Windsor \\ Eliza Ramsay \\ University of Windsor \\ Sara Scharoun Benson \\ University of Windsor
}

Follow this and additional works at: https://scholar.uwindsor.ca/humankineticspub

Part of the Kinesiology Commons

\section{Recommended Citation}

Salters, Danielle; Rios, P. Camila; Ramsay, Eliza; and Scharoun Benson, Sara. (2020). Preferential reaching and End-State Comfort: How task demands influence motor planning.. Journal of Motor Behavior, Online ahead of print.

https://scholar.uwindsor.ca/humankineticspub/41

This Article is brought to you for free and open access by the Faculty of Human Kinetics at Scholarship at UWindsor. It has been accepted for inclusion in Human Kinetics Publications by an authorized administrator of Scholarship at UWindsor. For more information, please contact scholarship@uwindsor.ca. 
Running Head: PREFERENTIAL REACHING AND END-STATE COMFORT

\section{Preferential Reaching and End-State Comfort: How Task Demands Influence Motor}

Planning

N9B3P4

Citation: Salters, D., Rios, P.C., Ramsay, E., \& Scharoun Benson, S. M. (2020). Preferential reaching and End-State Comfort: How task demands influence motor planning. Journal of Motor Behavior. https://doi.org/10.1080/00222895.2020.1858746

Sara M. Scharoun Benson, Ph.D., Assistant Professor Department of Kinesiology, University of Windsor 401 Sunset Ave, Windsor, Ontario, Canada, N9B3P4 sara.scharoun@uwindsor.ca|519.253.3000x.4994

\section{Word Count (Manuscript Body): 6501}

Word Count (Abstract): 120

Table Count: 1

Figure Count: 7 


\section{Abstract}

43 Various factors (e.g., hand preference, object properties) constrain reach-to-grasp in

44 hemispace. With object use, end-state comfort (ESC) has been shown to supersede the

45 preferential use of one hand at the midline. To assess how location, size, and orientation of

46 objects (dowel, mallet, cup) influence preferred-hand use and ESC (N=50; Mage = 20.83), three

47 preferential reaching tasks were implemented. Object location influenced hand selection in all

48 tasks, along with size (cups) and orientation (mallets). Object location and orientation influenced

49 ESC, but only with dowels and mallets. When oriented away from the preferred hand in

50 hemispace, there was a higher occurrence of non-preferred hand use to facilitate ESC. Overall,

51 findings add to understanding of ESC and preferential reaching with varying task demands.

52

53 Keywords: Motor Planning; End-State Comfort; Preferential Reaching; Hand Selection; Hand

54 Preference; Grasp Selection; Object Location; Object Orientation 


\section{Introduction}

Human performance of complex, multi-articular tasks can be explained by ecological

57 dynamics, where coordination and control are derived from the relationship between intentions,

58 perceptions, and actions. This view of motor skill performance purports that the relationship

59 between movement pattern stability (i.e., robustness of motor skills during

60 disturbances/perturbations) and flexibility (i.e., ability to adapt) is continually constrained in a

61 movement system (Araújo, Davids, \& Passos, 2013; Davids et al., 2012; Seifert, Komar, Araújo,

$62 \&$ Davids, 2016).

63 According to Gibson (1979), affordances are action opportunities based on individuals'

64 perception of the environment; they are specified in a frame of reference unique to the action

65 capabilities of each individual (Borghi \& Riggio, 2015; Gibson, 1979; Lamb et al., 2017; Seifert,

66 Komar, Araújo, \& Davids, 2016). In reaching and grasping, coordination both between and

67 within components of perception and action systems must be addressed (Kelso, 1995; Seifert,

68 Komar, Araújo, \& Davids, 2016). Movement selection is driven by the tendency to optimize

69 certain criterion and exploit affordances within the environment, which minimizes the cost

70 functions related to motor redundancy (Dounskaia \& Shimansky, 2016) and provides flexibility

71 of control (Dounskaia \& Wang, 2014). Adaptive behaviours reflect flexibility to guarantee

72 functional solutions to constraints, revealing stability against perturbations; stable movement

73 systems are characterized by reproducible and consistent movement patterns over time (Seifert,

74 Komar, Araújo, \& Davids, 2016). Individuals exploit inherent affordances and task constraints in

75 the movement system to achieve task goals (Seifert, Komar, Araújo, \& Davids, 2016).

Hand selection during reaching tasks is a function of the level of control that can be

77 attained by one hand compared to the other (Leconte \& Fagard, 2006; Stins, Kadar, \& Costall, 
2001). According to the motor dominance hypothesis, if hand preference exists in motor planning and execution, then the preferred hand will be used to perform most unimanual tasks (Bryden et al., 1994; Scharoun, Scanlan, \& Bryden, 2016). The preferred limb system achieves the most accuracy as a result of predictive control of limb and task dynamics (Pryzbyla et al., 2013; Sainburg, 2002); therefore, when both hands are in use, the preferred hand is chosen more

83 often for precise control of movement and manipulation of an object, as the non-preferred hand 84 is more adept at positioning.

When reaching into different regions of hemispace, the Kinesthetic Hypothesis outlines that biomechanical constraints on the preferred hand will lead to non-preferred hand use,

87 especially in contralateral space, favouring proximity of the object and movement efficiency

88 (Bryden \& Huszcynski, 2011; Gabbard \& Rabb, 2000; Rezae et al., 2010; Scharoun, Scanlan, \& 89 Bryden, 2016). The Kinesthetic Hypothesis, also referred to as the "comfort hypothesis"

90 (Gabbard \& Rabb, 2000, p. 181), argues that an individual does not necessarily consider an

91 object's location in hemispace, but will perceive the biomechanical constraints involved in the

92 task to perform the task with the most efficient and comfortable response; the key factor for the

93 Kinesthetic Hypothesis is that individuals will choose a movement based on their perception of

94 the actions afforded to them, and choose the most efficient of those actions. (Gabbard \& Rabb,

95 2000). Conversely, the Hemispheric Bias Hypothesis outlines the tendency for individual to use

96 each hand in ipsilateral space in order to accommodate spatial compatibility (Bishop, Ross,

97 Daniels, \& Bright, 1996; Bryden \& Huszcynski, 2011; Gabbard \& Rabb, 2000; Leconte \&

98 Fagard, 2004; Scharoun, Scanlan, \& Bryden, 2016). This bias reflects the ability of the hands to

99 perform faster and more accurately in ipsilateral space; where each hand will perform better in

100 its own region of hemispace (e.g., right hemispace-right hand, left hemispace- left hand). In 
101 contralateral space, there is greater need for interhemispheric communication, which results in a 102 loss of movement efficiency (Bryden \& Roy, 2006; Gabbard \& Rabb, 2000). Furthermore,

103 reaching in ipsilateral space promotes optimal visual processing within a single visual hemifield

104 (Leconte \& Fagard, 2006). Both the Kinesthetic Hypothesis and the Hemispheric Bias

105 Hypothesis are critical to understanding goal-oriented reaching tasks, as spatial information for

106 reaching in hemispace is either driven by perceptions through the Kinesthetic Hypothesis or

107 controlled through the Hemispheric Bias Hypothesis (Gabbard \& Rabb, 2000).

108 The spatial location of the target object is a fundamental constraint on hand selection

109 (Stins, Kadar, \& Costall, 2001). Perihand space boundaries influence action planning and

110 adjustments throughout the movement (Patane et al., 2018). In support of both the Kinesthetic

111 and Hemispheric Bias Hypotheses, research has demonstrated the preferred hand is used almost

112 exclusively in ipsilateral space, and less often in contralateral space (Bryden, Mayer, \& Roy,

113 2011); there is a bias to use each hand in its own region of hemispace (i.e., ipsilateral space)

114 where performance is both faster and more accurate than in contralateral space (i.e., reaching

115 across the midline; Bryden \& Roy, 2006; Coelho et al., 2013). A shift to the use of the non-

116 preferred hand is demonstrated in contralateral space (Coelho et al., 2013; Helbig \& Gabbard,

117 2013; Leconte \& Fagard, 2004; Leconte \& Fagard, 2006; Rezae et al., 2010; Scharoun, Scanlan,

$118 \&$ Bryden, 2016). General findings have outlined that adults use the preferred hand most often in

119 ipsilateral and midline reaches, although those with a strong right hand preference are likely to

120 continue to use the preferred hand in contralateral space, despite the potential biomechanical

121 inefficiencies (Bryden \& Huszczynski, 2011; Mamolo et al., 2006).

122 When people demonstrate a strong hand preference, this hand tends to be used in a larger

123 area in hemispace to accomplish a variety of tasks; when an individual is considered strongly 
124 lateralized, they are highly consistent in their hand choice for tasks, while less strongly

125 lateralized individuals are more likely to use their hands interchangeably to complete various

126 tasks (Stins, Kadar, \& Costall, 2001). Object characteristics also influence hand selection

127 (Bryden, Mayer, \& Roy, 2011). The use of tools has been shown to elicit greater preferred hand

128 selection compared to objects that may be perceived as ecologically irrelevant, where the use of

129 the tool leads to increases in preferred hand selection compared to grasping tasks (Bryden et al.,

130 2011; Mamolo, Roy, Rohr, \& Bryde, 2006; Scharoun, Scanlan, \& Bryden, 2016). There is also

131 an increase in preferred hand use when precision is needed for grasping and manipulation of the

132 object, related to task complexity (Stone, Bryant, \& Gonzalez, 2013). That said, people are more

133 likely to choose the hand that matches the alignment of the object (for example, if the handle of a

134 mug was oriented to the left, the individual would be more likely to choose their left hand)

135 regardless of the starting position of the hands (Bryden \& Huszczynski, 2011; Scharoun,

136 Scanlan, \& Bryden, 2016).

137 When manipulating the object is the goal, the movement is initiated in such a way that

138 anticipates the future demands of that manipulation. The grasp used reflects planning for actions

139 that contribute to aiming and precision (Herbort \& Kunde, 2019; Rosenbaum \& Sauerberger,

140 2019). First order planning involves consideration immediate task demands. In reach-to-grasp,

141 this involves shaping grip aperture based on perceptual effects of object properties. The term

142 micro-affordance has been used to describe how object characteristics (e.g., size, orientation)

143 evoke a specific motor response (Ellis \& Tucker, 2000). For example, Hermsdörfer and

144 colleagues (2006) described the performance of individuals with apraxia to improve with actual

145 tools compared to in pantomime, as "the affordances provided by the tool and the object may

146 evoke a particular motor representation" (p. 1642). 
Second-order planning, in comparison, includes consideration of both immediate and

148 subsequent task demands, dependent upon task complexity and planning requirements

149 (Rosenbaum et al., 2012). Second-order planning thus allows the individual to modify their

150 behaviour in anticipation of secondary task demands (Rosenbaum et al., 2012; Scharoun,

151 Gonzalez, Roy, \& Bryden, 2018). According to the precision hypothesis, individuals favour

152 grasps that promote end-state comfort, where having control at the end of the movement is

153 critical, and assumes that the control requirements for placing an object are higher than those for

154 grasping and/or lifting (Coelho, Studenka, \& Rosenbaum, 2014; Herbort \& Kunde, 2019;

155 Rosenbaum et al., 1990; Rosenbaum et al., 1993; Rosenbaum \& Sauerberger, 2019; Seegelke \&

156 Weigelt, 2018). The selected grasp for object manipulation is dependent upon the amount of

157 control needed at various points throughout the movement; an object is typically grasped

158 efficiently if control was needed at the beginning phase of the movement, and awkwardly if

159 control is needed towards the end of the movement (Rosenbaum \& Sauerberger, 2019).

160

From this perspective, it has also been argued that hand preference may be a malleable

161 feature in movement control and may be less important compared to other performance

162 variables, such as end-state comfort in grasping (Coelho, Studenka, \& Rosenbaum, 2014).

163 Support for this claim is derived from Coelho and colleagues (2014), who examined the bar-

164 transport task in two conditions: (1) designated hand (left or right), with freedom to select initial

165 grasp (overhand or underhand), and (2) designated initial grasp, with freedom to select hand.

166 Participants prioritized ending in a comfortable position over using the preferred hand; therefore,

167 indicating that end-state comfort was weighted as a greater constraint in action control. It is

168 important to note that this research observed object manipulation at the midline. 
Overall, it is understood that numerous factors influence the decision to select one hand (or

170 the other) when reaching to grasp and manipulate objects in regions of hemispace (e.g., object

171 location, object characteristics). Literature also reports that, when there exists an intention for

172 use, objects are grasped in consideration of both immediate and secondary requirements, and

173 thus result in a comfortable end-state posture. In such cases, the desire to end comfortably has

174 been shown to supersede the preferential use of one hand. However, literature to date has

175 generally examined the end-state comfort effect when object manipulation occurs at the midline.

176 In such cases, object properties, such as size and orientation, often remain consistent (i.e., are not

177 manipulated as a factor of inquiry). To garner additional insights regarding the interplay of hand

178 preference and second-order planning when objects are located in different regions of hemispace,

179 the current research assessed end-state comfort (i.e., trials where participants ended in a neutral

180 posture that facilitates object use without further manipulation) and hand selection (i.e., preferred

181 hand use within each task) within three preferential reaching tasks. Manipulations of object

182 orientation (dowel/mallet handle) and size (cup) were included to garner a better understanding

183 of how object properties and action intentions influence hand and grasp selection.

184 First, overturned cups were placed in five regions of hemispace; participants were asked to

185 pick up a cup and pretend to pour water from a pitcher located at the midline. To examine the

186 influence of object size, four sizes of cups were included. Subsequently, dowels (painted half

187 black) and rubber mallets were placed in three regions of space; participants were required to: (1)

188 pick-up and place a dowel on a tracing of the dowel at the midline, matching according to colour;

189 and (2) pick-up a rubber mallet and hit a nail in a wooden block at the midline. To delineate

190 whether orientation is influential, dowels and rubber mallets were oriented towards and away

191 from, and to the left and right of the participant. Dependent measures included the proportion of 
192 trials performed with preferred hand use and end-state comfort (i.e., ending a reach-to-grasp

193 movement in a neutral posture that facilitates object use without further manipulation).

194 It was hypothesized that a higher frequency of preferred hand selection would be displayed

195 in ipsilateral space and at the midline, but that the non-preferred hand would be used more often

196 in contralateral space; this hypothesis is in support of the kinesthetic hypothesis and hemispheric

197 bias hypothesis, where an individual is more likely to use each hand in their own region of

198 hemispace based on biomechanical constraints and spatial information (for example: Bryden \&

199 Huszczynski, 2011; Gabbard \& Rabb, 2000; Scharoun, Scanlan, \& Bryden, 2016). It was also

200 hypothesized that participants would demonstrate end-state comfort regardless of object location,

201 size, or orientation; characterized by the finding that initial grasps would be uncomfortable or

202 awkward to facilitate comfortable end-state postures (for example: Herbort \& Kunde, 2019;

203 Rosenbaum et al., 1990; Rosenbaum \& Sauerberger, 2019; Seegelke \& Weigelt, 2018). Finally,

204 it was expected that participants would prioritize a grasp that facilitated end-state comfort across

205 regions of space, over the decision to use the preferred hand (i.e., demonstrate end-state comfort,

206 albeit with the non-preferred hand). This hypothesis also supports the kinesthetic hypothesis

207 (Gabbard \& Rabb, 2000), where an individual will use the non-preferred hand based on

208 perceived biomechanical inefficiencies (for example: Bryden \& Huszczynski, 2011; Helbig \&

209 Gabbard, 2013; Leconte \& Fargard, 2006).

Methods

\section{Participants}

212 A convenience sample of 50 young adults between the ages of 18- and 26-years-old

213 (Mage $=20.83, \mathrm{SD}=1.78 ; 26$ Female, 24 Male; 45 right-handers, 5 left-handers) from the

214 undergraduate and graduate student body agreed to participate. All participants were without any 
215 known sensorimotor or cognitive impairments that may have influenced participation. To

216 confirm eligibility, a statement regarding inclusion/exclusion criteria, which included a detailed

217 list of disabilities and disorders that would disqualify the participant, was included in the

218 information letter. Written informed consent was obtained, indicating that the participant: (a) was

219 willing to participate in the research, and (b) permitted video recording.

220 Procedures

221 This research was reviewed and received ethics clearance from the University of Windsor

222 research ethics board (REB\#: 17-245). Data collection occurred in a laboratory space on the

223 university campus. Participants were seated across from a researcher for the duration of data

224 collection, which included a Waterloo Handedness Questionnaire (WHQ; Bryden, 1977), and a

225 series of three preferential reaching tasks with overturned cups, dowels, and rubber mallets

226 (Figure 2). The entire protocol lasted approximately 45 minutes. A video recorder was used to

227 record performance within each individual task; videos were coded offline. Unfortunately, some

228 of the video files were corrupted during file transfer; therefore, while all 50 participants

229 completed the three tasks, we were unable to code all videos. The number of participants is

230 stated in the data analysis section.

231 The WHQ asks participants to reflect on which hand they would use in a variety of

232 unimanual tasks. The 20-item version was used in the current research. Response options include

233 left always (-2), left usually, equal, right usually, and right always. Items are scored from -2 (left

234 always) to +2 (right always), allowing for a total score (range: $+/-40$ ) to be computed. It is

235 expected that right-handers have positive scores, whereas left-handers have negative scores.

236 Scores from the WHQ can be also be used to identify the degree or strength of hand preference.

237 For example, a score of +40 indicates a person strongly prefers the right hand; whereas a positive 
238 score closer to zero, such as +4 , indicates a weak right hand preference (Steenhuis \& Bryden, 239 1989).

240 In the first preferential reaching task (Figure 1A), 5 overturned cups were placed in front of 241 the participant at 0-, 45-, 90-, 135-, and 180-degrees (i.e., far left, near left, midline, near right, 242 far right). Glasses were identical in size and shape, but differing in colour, to allow the

243 researchers to refer to each cup by colour instead of location. A pitcher, with no handle, was 244 located at the participant midline. Participants were asked to pick up a designated cup and 245 demonstrate the action of pouring into the cup with the pitcher. To prevent instructions from 246 guiding performance, nothing else was specified. It is important to note that the overturned glass

247 task has revealed consistent results when implemented both with (Scharoun \& Bryden, 2014) and 248 without water (i.e., as if to pour water, e.g., Scharoun, Gonzalez, et al., 2016). Participants 249 complete three trials with four cup sizes $(4.7 \mathrm{~cm}, 6.2 \mathrm{~cm}, 7.2 \mathrm{~cm}$, and $8.4 \mathrm{~cm}$ diameter; Figure 2), 250 for a total of 60 trials. Trials were blocked by cup size; however, locations were randomized. 251 Dependent measures included the proportion of trials with preferred hand selection and end-state 252 comfort (i.e., ended the movement with a thumb-up grasp, with the wrist in a neutral position, 253 and the cup reoriented for use).

259 left, midline, right). Objects were identical in size and shape, but each had a coloured band to 260 allow the researchers to refer to each by colour instead of location. Participants were asked to 261 pick up a dowel and place it on a designated location, such that the orientation of the dowel 
matched a tracing of the dowel at the participants midline. Likewise, participants were asked to

263 pick up a mallet and hit a nail in a wooden block at the midline using the black side of the mallet

264 head. In both tasks, nothing else was specified, to prevent instructions from guiding

265 performance. Participants completed three trials of each task with four orientations (black end of

266 dowel/ mallet handle: towards, left, away, and right) for a total of 48 trials/task. Trials were

267 blocked by handle orientation; however, the locations were randomized. Dependent measures

268 included the proportion of trials with preferred hand selection and end-state comfort (i.e., ended

269 the movement with the wrist in a neutral position to place the dowel or hit the nail).

270 Data Analysis

271 SPSS $\odot$ statistical software (version 25) was used for data analyses. For the preferential

272 reaching task with overturned cups, the two dependent measures (1: proportion of trials with

273 preferred hand selection; and 2: proportion of trials with end-state comfort) were submitted to a

274 location (5: far right, near right, midline, near left, far left) by cup size $(4: 4.7 \mathrm{~cm}, 6.2 \mathrm{~cm}, 7.2 \mathrm{~cm}$,

$2758.4 \mathrm{~cm}$ diameter) within-subject analysis of variance test with repeated measures. The Bonferroni

276 correction was used for multiple comparisons. Data from 46 participants (Table 1) were included

277 in analysis, 5 of whom (i.e., $10.87 \%$ ) were left-handed (WHQ score $=-25.80, \mathrm{SD}=7.56$ ) and the

278 remaining 41 right-handed $(\mathrm{WHQ}$ score $=27.07, \mathrm{SD}=7.04)$. Although representative of the

279 general population, as right- and left-handed samples were not comparable in size, hand

280 preference was not included as a between subjects' factor; therefore, separate analyses of the

281 proportion of trials with preferred hand selection were performed for right- and left-handers. All

282 participants were included in analysis of end-state comfort. 

both tasks the two dependent measures (1: proportion of trials with preferred hand selection; and 2: proportion of trials with end-state comfort) were submitted to a location (3: right, midline,

287 left) by handle orientation (4: towards participant, left, away from participant, right) withinsubject analysis of variance test with repeated measures. The Bonferroni correction was used for 289 multiple comparisons. As the task was inherently different for left-handers, due to the orientation 290 of the objects, only data from 43 right-handed participants $\left(\mathrm{M}_{\mathrm{age}}=20.68, \mathrm{SD}=1.62 ; 23\right.$ Female, 29120 Male; WHQ Score $=27.47, \mathrm{SD}=6.93)$ were analyzed.

\section{Results}

Proportion of trials with preferred hand selection.

Overturned cups. Analysis of right-handed participants revealed a significant interaction

295 between size and location $\left(F(12,480)=2.740, p=.001, \eta^{2} p=.064\right.$; Figure 3). Pairwise

296 comparisons with a Bonferroni correction revealed that, for all cup sizes, there was no difference 297 in preferred (i.e., right) hand selection in near and far locations (both right and left); however, all 298 other locations were significantly different. In far right space, less preferred hand selection was 299 displayed with the $6.2 \mathrm{~cm}$ cup compared to the $7.2 \mathrm{~cm}$ cup. In far left space, less preferred hand 300 selection was seen with the 6.2 cup compared to the $8.4 \mathrm{~cm}$ cup. Finally, at the midline, 301 significantly less preferred hand selection was demonstrated with the $7.2 \mathrm{~cm}$ cup compared to the $3024.7 \mathrm{~cm}$ and $8.4 \mathrm{~cm}$ cups. The main effect of location $\left(F(4,160)=141.145, p<.001, \eta^{2}{ }_{p}=.779\right.$;

303 Figure 4) was consistent with the interaction. The effect of size was not significant $(F(3,120)=$ $\left.3042.422, p=.069, \eta^{2} p=.057\right)$. Analysis of left-handed participants identified a main effect of 305 location $\left(F(4,16)=14.035, p<.001, \eta^{2}=.778\right.$; Figure 4); however, when adjusted for multiple 306 comparisons using a Bonferroni correction, no differences were identified ( $p>.05)$. Neither the 
307 effect of size $\left(F(3,12)=1.400, p=.291, \eta^{2} p=.259\right)$ nor interaction between location and size

$308 \quad\left(F(12,48)=1.391, p=.203, \eta^{2}{ }^{2}=.258\right)$ were significant.

309

310

311

312

313

314

315

316
[Insert Figure 3 about here]

[Insert Figure 4 about here]

Dowels. A significant effect of location $\left(F(2,86)=80.687, p<.001, \eta^{2} p=.652\right)$ revealed significantly greater preferred-hand (i.e., right-hand) selection in right-space $(M=2.824, S E=$ $0.050)$ compared to the midline $(M=2.449, S E=0.111)$ and left-space $(M=1.063, S E=0.152)$.

The effect of orientation $\left(F(3,129)=2.381, p=.073, \eta^{2} p=.052\right)$ and interaction between orientation and location $\left(F(6,258)=.300, p=.936, \eta^{2} p=.007\right)$ were not significant.

Rubber Mallets. The interaction between location and orientation was significant $(F$ ( 6 , $258)=19.654, p<.001, \eta^{2} p=.319$; Figure 5), along with main effects of location $(F(2,86)=$ $\left.35.885, p<.001, \eta^{2}=.461\right)$ and orientation $\left(F(3,129)=36.975, p<.001, \eta^{2} p=.468\right)$. In leftspace, there was greater preferred-hand selection when the handle was oriented to the right and away from the participant, compared to when it was oriented to the left and towards the participant $(p<.001)$. At the midline and in right-space, the frequency of preferred-hand selection was lowest when the handle was oriented to the left compared to all other orientations $(p<.001)$. Also, in right-space, when the handle was oriented away from the participant, less preferred-hand selection was demonstrated compared to when oriented towards and to the right of the participant $(p=.024)$. When the handle was oriented towards the participant, to the left or right, significantly less preferred-hand selection was demonstrated in left-space compared to the midline and right-space $(p<.05)$. When the handle was oriented away from the participant, the frequency of preferred-hand selection was lower in left-space compared to right-space $(p=.041)$. Overall, the frequency of preferred-hand selection was highest in right-space compared to the 
331 midline and left-space $(p<.003)$; likewise, when the handle was oriented to the right and away

332 from the participant, compared to when oriented towards $(p<.001)$ and to the left $(p<.001)$.

334 Proportion of trials with end-state comfort

335 Overturned cups. Neither the interaction between size and location $(F(12,528)=1.342$, $\left.336 p=.190, \eta^{2}{ }^{2}=.030\right)$, nor the main effects of size $\left(F(3,132)=.702, p=.552, \eta^{2} p=.016\right)$ and

337 location $\left(F(4,176)=1.630, p=.169, \eta^{2} p=.036\right)$ were significant. It is important to note that the 338 frequency of end-state comfort was quite low in all regions of space (far right: $M=1.01, S E=$ 3390.17 , near right: $M=1.04, S E=0.17$, midline: $M=1.12, S E=0.17$, near left: $M=1.11, S E=$ 3400.17 , and far left: $M=1.12, S E=0.17)$ ) compared to previous reports (e.g., Scharoun, Gonzalez, 341 et al., 2016).

342 Dowels. The interaction between orientation and location $(F(6,252)=20.554, p<.001$, $343 \eta_{p}^{2}=.329 ;$ Figure 6$)$ and main effect of orientation $\left(F(3,126)=61.661, p<.001, \eta^{2}=.595\right)$

344 were significant; however, the effect of location was not $\left(F(2,84)=.437, p=.647, \eta^{2}{ }_{p}=.010\right)$.

345 Overall, there was significantly more end-state comfort when the black end of the dowel was 346 oriented towards and to the right of the participant compared to when it was oriented away ( $p<$ $347.001)$, and in all orientations when compared to the left $(p<.001)$. In left space, there was 348 significantly more ESC displayed when oriented to the right and away from the participant 349 (which did not differ) compared to towards $(p<.05)$, and in all orientations compared to when 350 oriented to the left $(p<.001)$. At the midline and in right space, the number of trials with ESC 351 was greater when oriented towards and to the right of the participant (which did not differ) 352 compared to when oriented away and to the left $(p<.001)$. In right space, there was no 353 difference when oriented away and to the left of the participant; however, at the midline, more 
354 ESC was displayed when oriented away $(p<.001)$. When oriented towards the participant,

355 significantly more ESC was demonstrated in right space compared to the midline $(p=.009)$ and

356 left space $(p<.001)$. When oriented to the right, significantly more ESC was displayed in right

357 compared to left space $(p=.044)$. When oriented away from the participant, significantly more

358 ESC was demonstrated in left space compared to the midline and right space $(p<.001)$, and at

359 the midline compared to right space $(p=.001)$.

[Insert Figure 6 about here]

$\left(F(6,252)=7.038, p<.001, \eta^{2} p=.144 ;\right.$ Figure 7$)$, alongside a main effect of orientation $(F(3$, $\left.126)=24.823, p<=.001, \eta^{2} p=.371\right)$. Overall, significantly more ESC was displayed when the

364 handle was oriented towards or to the right (which did not differ) compared to away from and to 365 the left of the participant $(p<.001$; which did not differ). This was true regardless of the region 366 of space $(p<.05)$. When the handle was oriented to the right, significantly more ESC was 367 demonstrated at the midline and in right space compared to left space $(p=.021)$. When the 368 handle was oriented away from the participant, significantly more ESC was displayed in left 369 space compared to the midline and right space $(p<.001)$. The main effect of location came up 370 short of the cut off for statistical significance $\left(F(2,84)=3.018, p=.054 ., \eta^{2} p=.067\right)$.

\section{Discussion}

The current study assessed end-state comfort and preferred hand selection within three 374 preferential reaching tasks; specifically, to examine reaching patterns related to object location, 375 movement context, and task demands. Three main predictions were made: first, that use of the 376 preferred hand would occur more often at midline and ipsilateral hemispace, and the non- 
377 preferred hand more often in contralateral space, in support of the kinesthetic hypothesis and the

378 hemispheric bias hypothesis, wherein the participant will use their preferred hand based on

379 perceptions of spatial affordances and biomechanical constraints (Bryden \& Huszczynski, 2011;

380 Gabbard \& Rabb, 2000; Scharoun, Scanlan, \& Bryden, 2016). Such behaviours were considered

381 important, as this study examined reaching from three perspectives: a bimanual reaching task

382 (the overturned cups), a rotation task (the dowels), and a tool-based task (the rubber mallets);

383 these tasks were chosen as progression through the different tests increased the demand on the

384 use of the hands, which has been shown to elicit stronger hand preference ratings (Bryden,

385 Mayer, \& Roy, 2011; Scharoun, Scanlan, \& Bryden, 2016). Second, it was hypothesized that

386 participants would demonstrate ESC regardless of object location, orientation, or size; initial

387 grasping behaviours typically reflect the anticipation of future demands of the manipulation

388 (Herbort \& Kunde, 2019; Rosenbaum \& Saurberger, 2019). These demands favour end-state

389 comfort when there is increased precision necessary within the movement, and assumes that the

390 control requirements during these stages are higher than the requirements for grasping and/or

391 lifting of the object (Herbort \& Kunde, 2019). Finally, it was hypothesized that ESC would be

392 prioritized higher than hand selection (Coelho et al., 2014), further supporting approaches to

393 motor control and reaching in hemispace based on the kinesthetic hypothesis (Gabbard \& Rabb,

394 2000). To allow for specific discussion, the following section will discuss the overturned cup

395 task separately from the dowel and mallet tasks, followed by a general discussion.

396 Overturned Cups

397 Previous evidence for reaching and grasping during unimanual tasks in right-handed

398 individuals demonstrated a preference for the right hand when picking up objects at the midline

399 and in ipsilateral space, with increased likelihood of using the preferred hand in contralateral 
400 space according to increases in task complexity (e.g., Bishop et al., 1996; Bryden and Roy,

401 2006; Mamolo et al., 2004, 2006; Scharoun, Scanlan, \& Bryden, 2016). Results from the

402 overturned cup task provide further evidence in support of hand selection based on object

403 location in hemispace and task complexity.

404 Contradictory to previous research assessing ESC, which has demonstrated consistent

405 patterns of ESC planning in the overturned cup task (e.g., Scharoun Benson et al., 2018), this

406 pick-up and pour task with overturned cups demonstrated lower prevalence of ESC. There was a

407 greater tendency for participants to assume a comfortable grasp at the beginning of the

408 movement (e.g., hand covering the bottom of the cup; pinch between the thumb and first finger,

409 and/or thumb and first two fingers) and complete the movement with the wrist in an awkward,

410 supinated position. Fuelscher et al. (2016) found that there is a greater tendency for participants

411 to adopt initial grasps that facilitate ESC when movements were simple (one-step) compared to

412 more complex movements. As the cups task represented a multi-step movement, the results of

413 the current study support the finding that lower ESC may be observed (Fuelscher et al., 2016).

414 In the current study, the jug was not filled with water and participants were not required to

415 pour water into the cups, rather to mimic pouring and then return the cup to the overturned

416 position; completion of the task did not necessitate the consideration of ESC during the pouring

417 action (e.g., with the wrist in a neutral position), as the cup was being returned to the original

418 position at the end of the movement. This could be further explained by the quasi-pantomime

419 nature of the pick and pour task; pantomime tasks require greater cognitive processing, where an

420 individual relies on their mental representation of the action throughout the movement (Bhoryoo

421 et al., 2020; Fuelscher et al., 2016; Scharoun, Gonzalez, Roy, \& Bryden, 2018; Stöckel \& 
422 Hughes, 2016). By removing the actual water from the movement, participants are using

423 increased working memory to mimic manipulation of the jug and cups throughout the task.

424 The current results support the finding that individuals tend to grasp objects based on the

425 intended action; where bimanual performance is constrained by the intended goal states

426 (Seegelke \& Weigelt, 2018). The participants grasped the cups based on the intention to return

427 the cup to an overturned position, rather than the pouring action that was analyzed. There was a

428 division of labour between the hands; the more an individual uses their preferred hand for

429 manipulation and grasping tasks, the more they use the non-preferred hand for stabilization

430 during those tasks (Coelho et al., 2013; Guiard, 1987; Pryzbyla et al., 2013; Scharoun, Scanlan,

431 \& Bryden, 2016; Stone, Bryant, \& Gonzalez, 2013). Participants in the current study were

432 required to manipulate the pitcher and cup by mimicking a pouring action with the empty jug.

433 The movement required third-order planning (i.e., pick-up, pour, place) in order to accurately

434 perform the task of holding the pitcher with one hand, picking up and supporting the cup with the

435 other, and then returning the cup to an overturned position. It was expected that this task would

436 represent second-order planning of movements (i.e., pick-up, pour), however, as participants

437 were not required to pour water into the cup, the mimicking task influenced the initial grasp of

438 the overturned cup. While the expected second-order planning allows the individual to plan

439 behaviours based on the secondary task demands, participants in this study unexpectedly planned

440 movement based on the tertiary demands of the movement (replacing the cup after the pouring

441 action; see also Rosenbaum et al., 2012; Scharoun, Scanlan, \& Bryden, 2016).

\section{Dowels and Rubber Mallets}

443 As expected, examination of hand selection and location in the dowel task revealed data

444 that are congruent with previous research findings. The dowel task represented a pick-up and 
445 place task combined with a rotation task, characterized by the need to rotate the dowel to ensure

446 proper placement on the place marker. When the black end of the dowel was oriented opposite

447 the place marker, participants adopted creative grasp and rotation solutions in order to assume

448 ESC. Both the dowel and mallets task results are consistent with the finding that when engaged

449 in object rotation tasks, participants will employ motor imagery techniques to mentally represent

450 the movement to be completed and generate a forward model of the action (Barhoun et al., 2019)

451 During both the mallet and dowel tasks, the ending position with ESC (placing the dowel

452 or hitting the nail) resulted in adopting a relatively similar neutral wrist position. This finding is

453 consistent with previous research which details that an individual demonstrates more ESC when

454 using the tool or object to complete a task (Bryden, Mayer, \& Roy, 2011; Mamolo et al., 2006;

455 Scharoun, Scanlan \& Bryden, 2016). Interestingly, the dowel task required further exploitation of

456 affordances than both the cups and the mallet tasks to account for rotation of the dowel to place it

457 appropriately with ESC (e.g., Barhoun et al., 2019). This finding is consistent with Herbort and

458 Kunde (2019), who found that when ESC was considered by participants there were improved

459 object translations, rotations, manipulation times, and movement errors throughout the execution

460 of the action.

461 As with previous research, object orientation influenced hand selection regardless of object

462 location in hemispace (Bryden \& Huszczynski, 2011; Chan \& Hoffman, 2012; Scharoun,

463 Scanlan, \& Bryden, 2016). The mallet task in the current study provides supporting evidence for

464 this trend in reaching and grasping literature. When the handle of the mallet was oriented away

465 from the participants' preferred hand (in left hemispace and handle oriented away and to the left)

466 the participant was more likely to use their non-preferred hand to reach for, and use the mallet to

467 hit the nail. In this task, participants were also less likely to demonstrate ESC when they used 
468 their non-preferred (left) hand. The combination of object orientation and hand selection with 469 ESC demonstrates a combined influence of preference and comfort in tasks that require a use 470 condition after reaching and grasping. Furthermore, the mallet task, in particular, supported

471 previous findings that reaching behaviours differed when affordances for action were detected by

472 the participants (Scharoun Benson, Bryden, \& Roy, 2019; Scharoun Benson, Roy, \& Bryden,

473 2018); mallets had clear handles that participants could afford a grasp to in order to hit the nail

474 with the rubber head of the mallet, while the cups and dowels did not have handles or other

475 markers that afforded a specific use (the coloured ends of the dowels were used to plan rotations

476 to hit the target, neither end had a specific use).

477 Compared to the overturned cup task, the dowel and mallet data demonstrated support for 478 previous research on preferential reaching and use of tools; when presented with a specific task, 479 the use of tools is likely to elicit greater use of the preferred hand, overriding the potential 480 biomechanical inefficiencies of reaching into contralateral space (Bryden, Mayer, \& Roy, 2011; 481 Scharoun, Scanlan, \& Bryden, 2016).

\section{General Discussion}

483 In the proportion of trials related to hand selection and preferential reaching (a reach and 484 grasp task), the data support the Kinesthetic Hypothesis, where object proximity and movement 485 efficiency are taken into account when planning movements (Gabbard \& Helbig, 2004).

486 Participants were not asked to grasp any of the cups, dowels, or mallets in specific ways for the 487 reach and grasp tasks; however, participants grasped the objects in ways that were congruent 488 with previous research on reaching and grasping in hemispace. Reaches at the midline and in 489 ipsilateral space were performed more often by the preferred hand, where reaches in contralateral 490 space were performed more often by the non-preferred hand (Bryden \& Huszczynski, 2011; 
491 Bryden, Mayer, \& Roy, 2011; Leconte \& Fagard, 2004; Leconte \& Fagard, 2006; Rezae et al., 492 2010).

493 Grasp selection for object manipulation combines habitual and goal-directed processing, 494 where the habitual system demonstrates a stronger influence on grasp selection; motor planning 495 for grasping tasks is most necessary when performing the movement for the first time, after 496 which the individual is likely to use recall of motor plans in the habitual system to perform the 497 task (Herbort, Mathew, \& Kunde, 2016). The current study further demonstrates the importance 498 of object location when determining hand selection in preferential reaching tasks; demonstrating 499 increased use of the non-preferred hand in contralateral space in reach and grasp tasks (Bryden \& 500 Huszczynski, 2011; Bryden, Mayer, \& Roy, 2011; Bryden, Pryde, \& Roy, 2000; Calvert \& 501 Bishop, 1998; Mamolo, Roy, Rohr, \& Bryden, 2006). Task demands have been identified as a contributing factor to reaching preferences, where 503 the greater the demand, the greater the likelihood of use of the preferred hand, even in 504 contralateral space (Bryden, Mayer, \& Roy, 2011; Cohelho, Studenka, \& Rosenbaum, 2014;

505 Steenhuis \& Bryden, 1999; Scharoun, Scanlan, \& Bryden, 2016). The intended action influences 506 how objects are handled, while the grasp posture at the onset of the movement can help to 507 determine how far in advance actions are planned (Scharoun, Gonzalez, Roy, \& Bryden, 2018).

508 In summary, the present study provides additional support for reaching and grasping 509 literature for unimanual tasks, where individuals will use their preferred hand more often in

510 contralateral space according to task demands and despite the potential biomechanical

511 inefficiencies. This study furthers existing literature on ESC in the dowel and mallet tasks

512 through evidence of increased use of the non-preferred hand according to object orientation;

513 participants used their non-preferred hand more readily when the handle was oriented away from 
514 the preferred hand, promoting biomechanical efficiency with grasping for these tasks. Finally,

515 the participants in this study demonstrated stronger evidence for third-order planning, compared

516 to second-order planning in the cup task. This is likely attributed to task complexity and

517 demands, where returning the objects to the start position was likely considered in the motor

518 planning phase. Hand selection for grasping and manipulation is a variable and task dependent

519 phenomenon; the task end-goal, task demands, biomechanical efficiency, and object location and

520 orientation in hemispace influence preferential reaching and grasping behaviours (Helbig \&

521 Gabbard, 2013; Gonzalez, Flindall, \& Stone, 2015). Together, the findings from the present

522 study add to the reaching and grasping literature with a consideration for ESC, and provide

523 further evidence for the influence of task demands on motor planning and execution in both

524 unimanual and bimanual tasks. It is, however, important to note that this study did include a 525 small number of left-handed participants, which is a clear limitation of this work. Future work 526 assessing the performance of left-handed participants is recommended.

\section{Disclosure Statement}

The authors have no conflict of interest to disclose.

\section{Data Availability Statement}

The data that support the findings of this study are available from the corresponding

531 author (SMSB), upon reasonable request. 


\section{References}

534

535

536

537

Araújo, D., Davids, K., Passos, P., 2013. The intending-perceiving-acting cycle in sports performance. In: McGarry, T., O’Donoghue, P., Sampaio, J. (Eds.), The Routledge Handbook of Sports Performance Analysis. Routledge, Taylor \&Francis Group, London, UK, pp. 32-41.

Barhoun, P., Fuelscher, I., Kothe, E. J., He, J. L., Youssef, G. J., Enticott, P. G., . . Hyde, C. (2019). Motor imagery in children with DCD: A systematic and meta-analytic review of hand-rotation task performance. Neuroscience and Biobehavioral Reviews, 99, 282-297.

Bhoyroo, R., Hands, B., Steenbergen, B., \& Wigley, C. A. (2020). Examining complexity in grip selection tasks and consequent effects on planning for end-state-comfort in children with developmental coordination disorder: A systematic review and meta-analysis. Child Neuropsychology, 26(4), 534-559. doi: 10.1080/09297049.2019.1695768

Bishop, D. V. M., Ross, V. A., Daniels, M. S., \& Bright, P. (1996). The measurement of hand preference: A validation study comparing three groups of right-handers. British Journal of Psychology, 87(2), 269-285. https://doi.org/10.1111/j.2044-8295.1996.tb02590.x

Borghi, M., \& Riggio, L. (2015). Stable and variable affordances are both automatic and flexible. Frontiers in Human Neuroscience, 9(351). doi: 10.3389/fnhum.2015.00351

Bryden, M. P. (1977). Measuring handedness with questionnaires. Neuropsychologia, 15(4-5), 617-624. https://doi.org/10.1016/0028-3932(77)90067-7

Bryden, M.P., Singh, M., Steenhuis, R.E., Clarkson, K.L. (1994). A behavioral measure of hand preference as opposed to hand skill. Neuropsychologia 32, 991-999. doi:10.1016/002839329490048-5 
555

Bryden, P.J., \& Huszczynski, J. (2011). Under what conditions will right-handers use their left hand? The effects of object orientation, object location, arm position, and task complexity in preferential reaching. Laterality, 16(6), 722-736. http://dx.doi.org/10.1080/1357650X.20 $\underline{10.514344}$

Bryden, P.J., Mayer, M., \& Roy, E.A. (2011). Influences of task complexity, object location, and object type on hand selection in reaching in left and right-handed children and adults. Developmental Psychobiology, 53(1), 47-58. DOI 10.1002/dev.20486

Bryden, P. J., Pryde, K. M., \& Roy, E. A. (2000). A performance measure of the degree of hand preference. Brain and Cognition, 44(3), 402-414. https://doi.org/10.1006/brcg.1999.1201

Bryden, P. J., \& Roy, E.A. (2006). Preferential reaching across regions of hemispace in adults and children. Developmental Psychobiology, 48(2), 121-132. DOI 10.1002/dev.20120

Bryden, M. P., Singh, M., Steenhuis, R., \& Clarkson, K. L. (1994). A behavioural measure of hand preference as opposed to hand skill. Neuropsychologia, 32(8), 991-999. https://doi.org/10.1016/0028-3932(94)90048-5

Calvert, G. A., \& Bishop, D. V. M. (1998). Quantifying hand preference using a behavioural continuum. Laterality: Asymmetries of Body, Brain and Cognition, 3(3), 255-268. DOI: $10.1080 / 713754307$

Chan, A.H.S., \& Hoffmann, E.R. (2012). Movement Compatibility for configurations of displays located in three cardinal orientations and ipsilateral, contralateral and overhead controls. Applied Ergonomics, 34(1), 128-140. https://doi.org/10.1016/j.apergo.2011.04.005

Coelho, C.J., Przybyla, A., Yadav, V., \& Sainburg, R.L. (2013). Hemispheric differences in the control of limb dynamics: a link between arm performance asymmetries and arm selection patterns. Journal of Neurophysiology, 103(3), 825-838. Doi:10.1152/jn.00885.2012. 
578 Coelho, C.J., Studenka, B., \& Rosenbaum, D.A. (2014). End-state comfort trumps handedness in object manipulation. Journal of Experimental Psychology: Human Perception and Performance, 40(2), 718-730. DOI: 10.1037/a0034990

Davids, K., Araújo, D., Hristovski, R., Passos, P., Chow, J.Y., 2012. Ecological dynamics and motor learning design in sport. In: Hodges, N.J., Williams, A.M.(Eds.), Skill Acquisition in Sport: Research, Theory and Practice. Routledge (Taylor and Francis Group), New York, NY, USA, pp. 112-130.

Dounskaia, N., \& Shimansky, Y. (2016). Strategy of arm movement control is determined by minimization of neural effort for joint coordination. Experimental Brain Research, 234, 1335-1350. DOI 10.1007/s00221-016-4610-z

Dounskaia, N., \& Wang, W. (2014). A preferred pattern of joint coordination during arm movements with redundant degrees of freedom. Journal of Neurophysiology, 112(5), 1040-

Ellis, R., \& Tucker, M. (2000). Micro-affordance: The potentiation of components of action by seen objects. British journal of psychology, 91(4), 451-471.

593 Fuelscher, I., Williams, J., Wilmut, K., Enticott, P. G., \& Hyde, C. (2016). Modeling the maturation of grip selection planning and action representation: Insights from typical and atypical motor development. Frontiers in Psychology, 7(108). doi:10.3389/fpsyg.2016.00108

Gabbard, C., \& Helbig, C. (2004). What drives children's limb selection for reaching in hemispace? Experimental Brain Research, 156, 325-332. https://doi.org/10.1007/s00221$\underline{003-1792-y}$ 
600 Gabbard, C., \& Rabb, C. (2000). What determines choice of limb for unimanual reaching movements? J. Gen. Psychol. 127, 178-184.doi: 10.1080/00221300009598577

602 Gibson, J.J. (1979). The Ecological Approach to Visual Perception. Boston: Houghton Mifflin.

603 Gonzalez, C. L., Flindall, J. W., \& Stone, K. D. (2015). Hand preference across the lifespan:

604 Effects of end-goal, task nature, and object location. Frontiers in Psychology, 5(1579).

$605 \quad$ https://doi.org/10.3389/fpsyg.2014.01579

606 Guiard, Y.(1987).Asymmetric division of labor in human skilled bimanual action: the kinematic chain as a model. J. Mot. Behav. 19, 486-517.doi: 10.1080/00222895.1987.10735426

608 Helbig, C.R., \& Gabbard, C. (2004). What determines limb selection for reaching? Research 609 Quarterly for Exercise and Sport, 75(1), 47-59. DOI:10.1080/02701367.2004.10609133

610 Herbort, O., \& Kunde, W. (2019). Precise movements in awkward postures: A direct test of the precision hypothesis of the end-state comfort effect. Journal of Experimental Psychology: Human Perception and Performance, 45(5), 681-696. http://dx.doi.org/10.1037/xhp0000639

614 Herbort, O., Mathew, H., \& Kunde, W. (2017). Habit outweighs planning in grasp selection for 615 object manipulation. Cognitive Psychology, 92, 127-140. https://doi.org/10.1016/j.cogpsyc $\underline{\text { h.2016.11.008 }}$

617 Hermsdörfer, J., Hentze, S., \& Goldenberg, G. (2006). Spatial and kinematic features of apraxic movement depend on the mode of execution. Neuropsychologia, 44(10), 1642-1652.

619 Kelso, J.A.S., 1995. Dynamic patterns: The self-organization of brain and behavior. MIT, 620 Cambridge, MA. 
621 Lamb, M., Kallen, R.W., Harrison, S.J., Di Bernardo, M., Minai, A., \& Richardson, M.J. (2017).

622 To pass or not to pass: Modeling the movement and affordance dynamics of a pick and 623 place task. Frontiers in Psychology, 8(1061). doi: 10.3389/fpsyg.2017.01061

624 Leconte, P., \& Fagard, J. (2004). Influence of object spatial location and task complexity on 625 children's use of their preferred hand depending on their handedness consistency. Developmental Psychobiology, 45(2), 51-58. DOI 10.1002/dev.20019

Leconte, P., \& Fagard, J. (2006). Which factors affect hand selection in children's grasping in hemispace? Combined effects of task demand and motor dominance. Brain and Cognition, 60(1), 88-93. doi:10.1016/j.bandc.2005.09.009

Mamolo, C.M., Roy, E.A., Rohr, L.E., \& Bryden, P.J. (2006). Reaching patterns across working space: The effects of handedness, task demands, and comfort levels. Laterality, 11(5), 465-

633 Patane, I., Cardinali, L., Salemme, R., Pavani, F., Farne, A., \& Brozzoli, C. (2018). Action planning modulates peripersonal space. Journal of Cognitive Neuroscience, 31(8), 11411154. doi:10.1162/jocn_a_01349

Przybyla, A., Coelho, C.J., Akpinar, S., Kirazci, S., \& Sainburg, R.L. (2013). Sensorimotor performance asymmetries predict hand selection. Neuroscience, 228(1), 349-360. http://dx.doi.org/10.1016/j.neuroscience.2012.10.046 affect hand selection in adults? Combined effects of ocular dominance, task demand and object location. Journal of Human Kinetics, 26, 39-44. DOI: 10.2478v10078-010-0046-x 
642 Rosenbaum, D.A., Chapman, K.M., Weigelt, M., Weiss, D.J., \& van der Wel, R. (2012). Cognition, action, and object manipulation. Psychol. Bull. 138(5), 924-946. doi: $10.1037 / \mathrm{a} 0027839$

Rosenbaum, D. A., Marchak, F., Barnes, H. J., Vaughan, J., Slotta, J., \& Jorgensen, M. (1990). Constraints for action selection: Overhand versus underhand grips. In M. Jeannerod (Ed.), Attention and Performance XIII: Motor representation and control (pp. 321-342).

Rosenbaum, D.A., \& Sauerberger, K.S. (2019). End-state comfort meets procrastination. Psychological Research, 83, 205-215. https://doi.org/10.1007/s00426-018-01142-6

Rosenbaum, D. A., Vaughan, J., Jorgensen, M. J., Barnes, H. J., \& Stewart, E. (1993). Plans for object manipulation. In D. E. Meyer \& S. Kornblum (Eds.), Attention and performance 14: Synergies in experimental psychology, artificial intelligence, and cognitive neuroscience (pp. 803-820). Cambridge: MIT Press, Bradford Books.

Sainburg, R. L. (2002). Evidence for a dynamic-dominance hypothesis of handedness. Experimental brain research, 142(2), 241-258. doi: 10.1007/s00221-0010913-8

Scharoun Benson, S. M., Bryden, P. J., \& Roy, E. A. (2019). Age-group differences in beginning-state comfort reveal an increase in motor planning capabilities. International Journal of Behavioral Development, 43(6), 563-568. doi: 10.1177/0165025419865620

661 Scharoun Benson, S. M., Roy, E.A., \& Bryden, P. J. (2018). End-state comfort in two object 662 manipulation tasks: Investigating how the movement context influences planning in children, young adults, and older adults. Developmental Psychobiology, 60(3), 317-323. doi: 10.1002/dev.21611 
665 Scharoun, S.M., \& Bryden, P.J. (2014). The development of end- and beginning-state comfort in a cup manipulation task. Developmental Psychobiology, 56(3), 407-420. doi:10.1002/dev.21108

668

Scharoun, S.M., Gonzalez, D.A., Roy, E.A., \& Bryden, P.J. (2016). How the mode of action affects evidence of planning and movement kinematics in aging: End-state comfort in older adults. Developmental Psychobiology, 58(4), 439-449. https://doi.org/10.1002/dev.21386

Scharoun, S.M., Gonzalez, D.A., Roy, E.A., \& Bryden, P.J. (2018). End-state comfort across the lifespan: A cross-sectional investigation of how movement context influences motor planning in an overturned glass task. Motor Control, 22(2), 211230. https://doi.org/10.1123/mc.2016-0064

Scharoun, S.M., Scanlan, K.A., \& Bryden, P.J. (2016). Hand and grasp selection in a preferential reaching task: The effects of object location, orientation, and task intention. Frontiers in Psychology, 7(360). doi: 10.3389/fpsyg.2016.00360

Seegelke, C., \& Weigelt, M. (2018). Anticipating different grips reduces bimanual end-state comfort: A tradeoff between goal-related and means-related planning processes. PLoS ONE, 13(1). https://doi.org/10.1371/journal.pone.0190586

Seifert, L., Komar, J., Araújo, D., \& Davids, K. (2016). Neurobiological degeneracy: A key property for functional adaptations of perception and action to constraints. Neuroscience and Biobehavioral Reviews, 69, 159-165. https://doi.org/10.1016/j.neubiorev.2016.08.006

Steenhuis, R. E., \& Bryden, M. P. (1989). Different dimensions of hand preference that relate to skilled and unskilled activities. Cortex, 25(2), 289-304. https://doi.org/10.1016/S00109452(89)80044-9 
687 Steenhuis, R. E. \& Bryden, M. P. (1999). The relation between hand preference and hand performance: What you get depends on what you measure. Laterality: Asymmetries of 689 Body, Brain and Cognition, 4(1), 3-26.

690 Stins, J.F., Katar, E.E., \& Costall, A. (2001). A kinematic analysis of hand selection in a reaching task. Laterality: Asymmetries of Body, Brain and Cognition, 6(4), 347-367. DOI: $10.1080 / 713754421$

693 Stöckel, T., \& Hughes, C.M. (2016). The relation between measures of cognitive and motor 694 functioning in 5- to 6-year-old children. Psychological Research, 80(4), 543-554.

695 doi:10.1007/s00426-015-0662-0

696 Stone, K.D., Bryant, D.C., \& Gonzalez, C.L.R. (2013). Hand use for grasping in a bimanual task:

697 Evidence for different roles? Experimental Brain Research, 224, 455-467. DOI

698 $10.1007 / \mathrm{s} 00221-012-3325-\mathrm{z}$ 
PREFERENTIAL REACHING AND END-STATE COMFORT

Table

Mean Age (SD) Sex (Female:Male) Mean WHQ (SD)

$\begin{array}{llll}\text { Total }(N=46) & 20.83(1.86) & 26: 20 & 21.33(18.06) \\ \text { Right-handers }(\mathrm{n}=41) & 20.78(1.82) & 24: 17 & 27.07(7.04) \\ \text { Left-handers }(\mathrm{n}=5) & 21.25(2.50) & 2: 3 & -25.80(7.56)\end{array}$

701

702 


\section{Table Caption}

704 Table 1. Participant demographics for preferential reaching task with overturned cups 705

706 
Figure 1

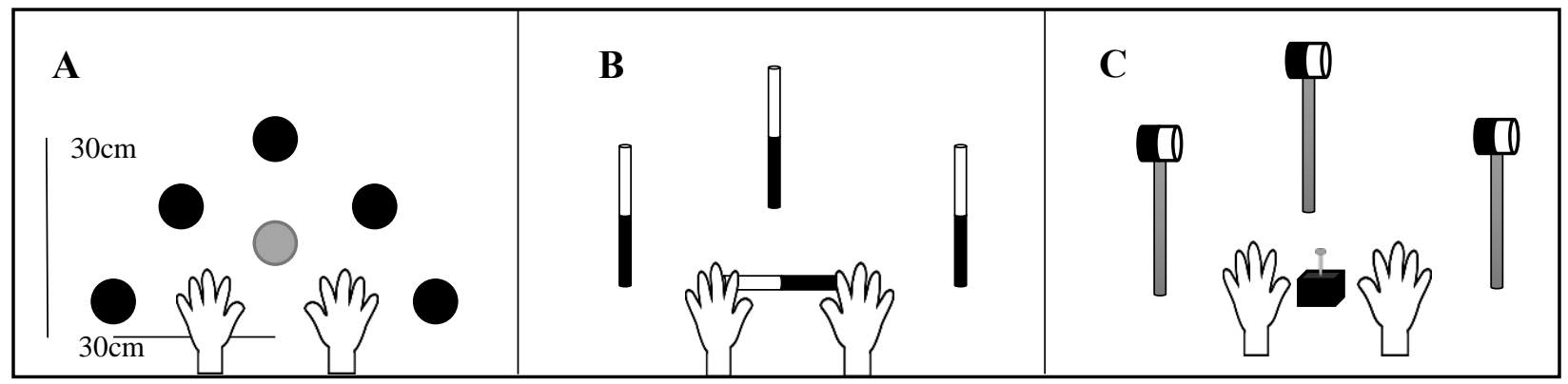

710

711

712

Figure 2

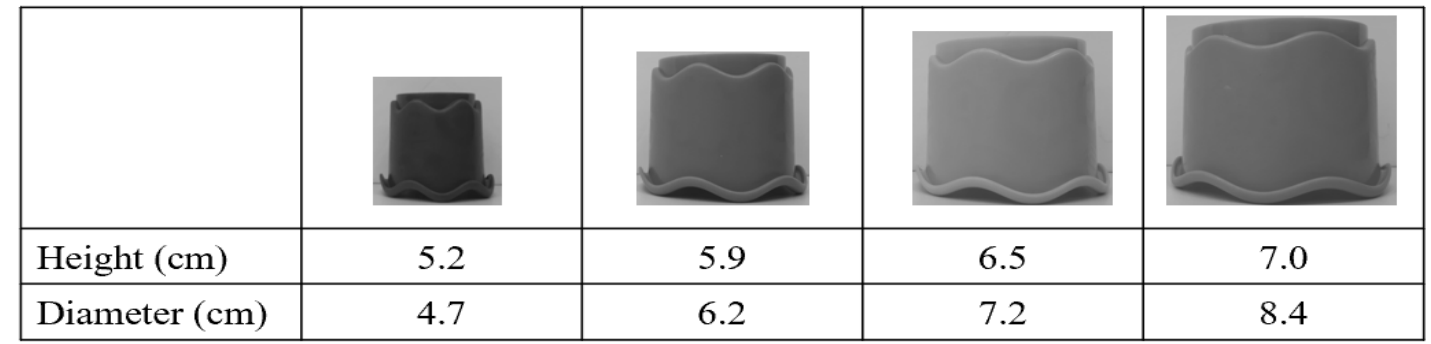

713

714

Figure 3

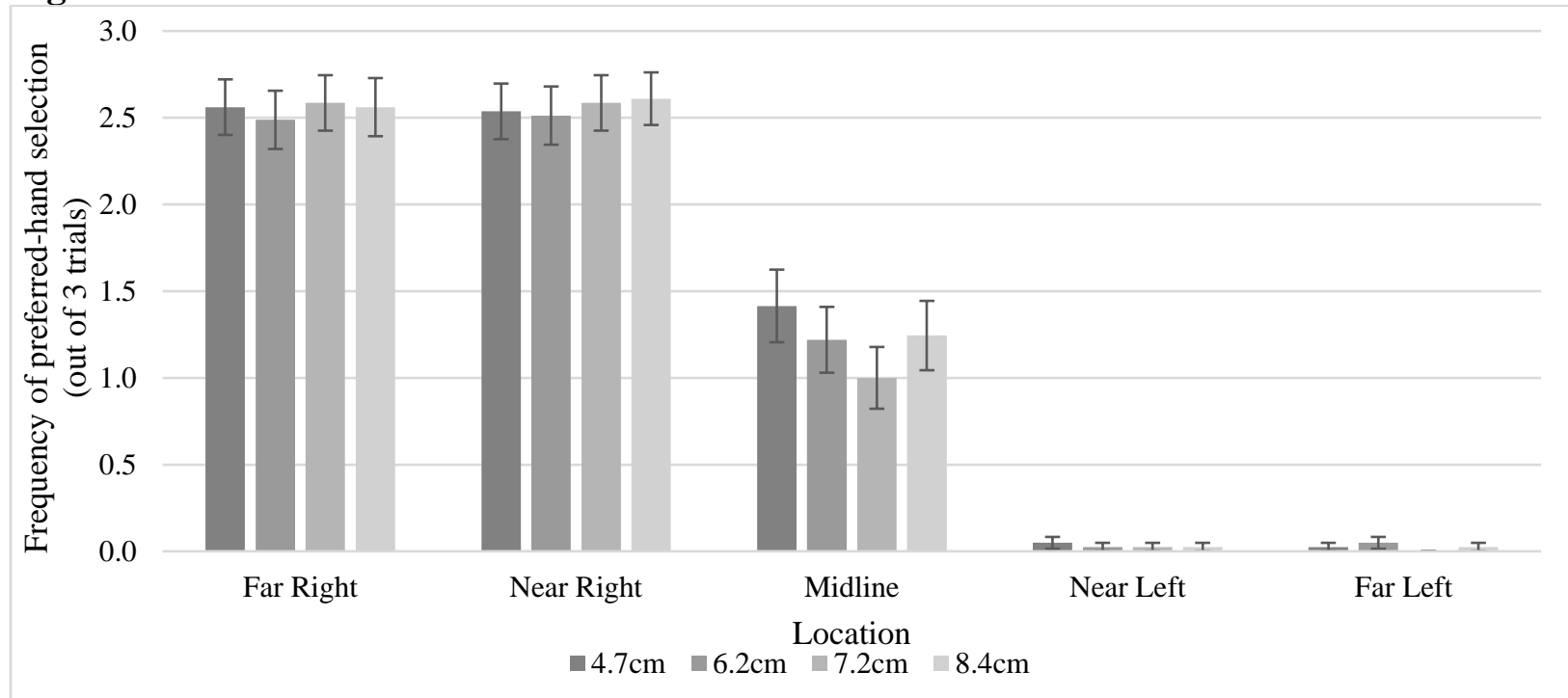


Figure 4

724

725

726

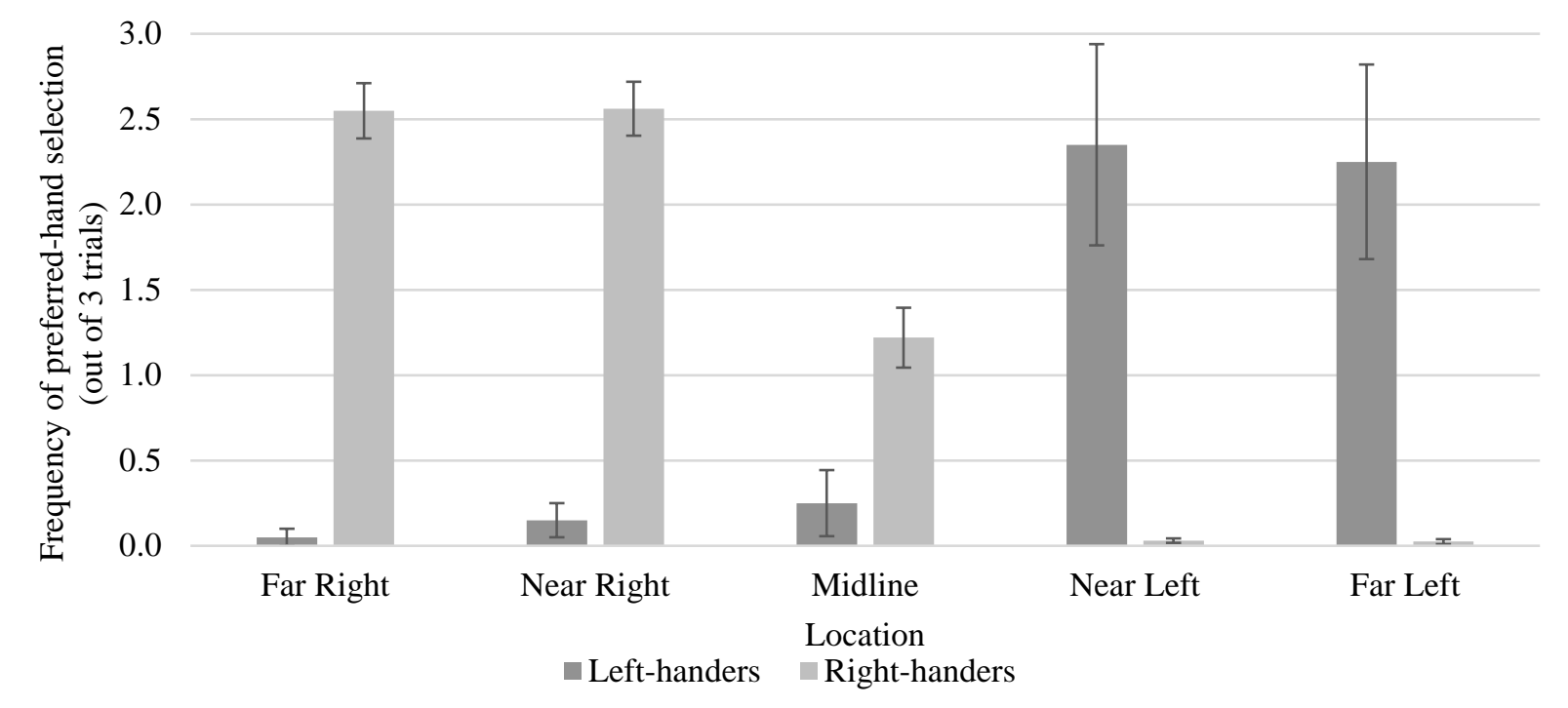

\section{Figure 5}

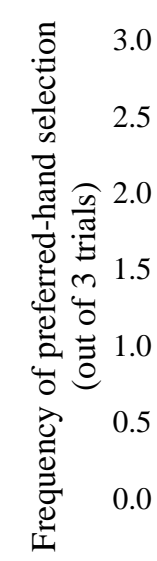

0.0
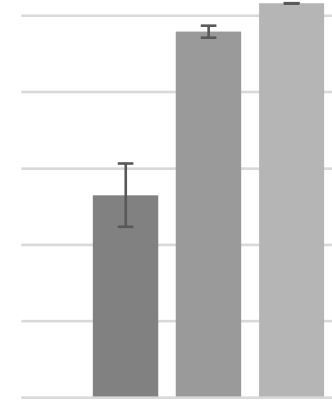

Left Space

Handle Towards
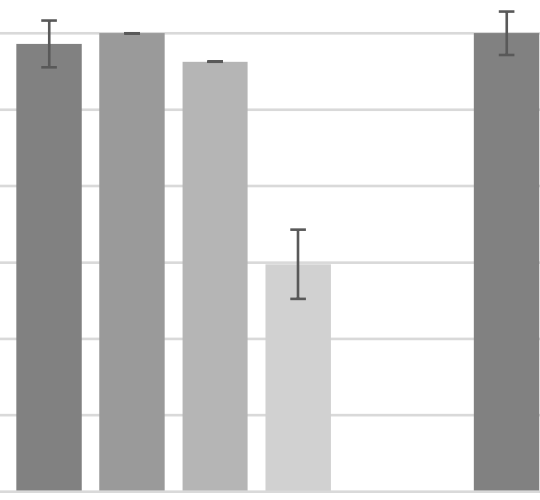

Midline

Location

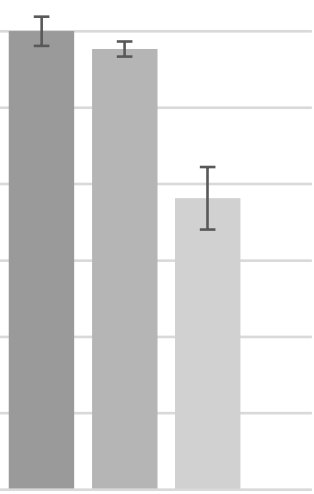

Right Space 
Figure 6

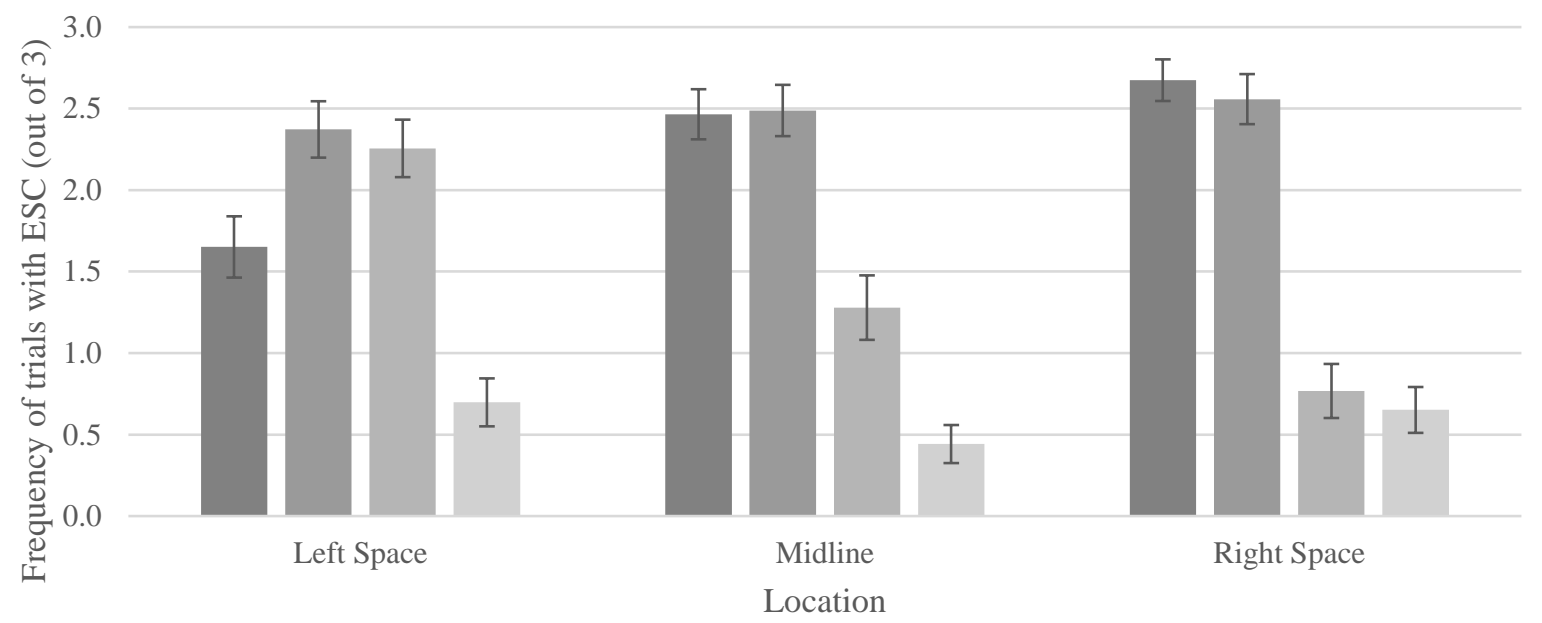

घ Towards $\quad$ Right $\square$ Away $\square$ Left

Figure 7

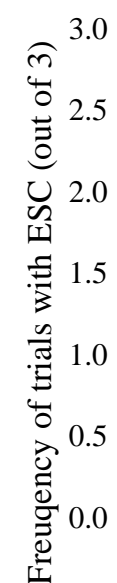

744

745

746 
Figure Captions

748 Figure 1. Study set up with: (A) Overturned cups, (B) Dowels, and (C) Rubber Mallets

749 Figure 2. Cup dimensions

750 Figure 3. Analysis of right-handers revealed a significant interaction between location and cup 751 size in analysis of preferred-hand selection with the overturned cups. Standard error bars are 752 displayed.

753 Figure 4. A main effect of location was revealed for both left- and right-handers in analysis of 754 preferred-hand selection with the overturned cups. Standard error bars are displayed.

755 Figure 5. Analysis of right-handers revealed a significant interaction between location and 756 orientation in analysis of preferred-hand selection with the rubber mallets. Standard error bars 757 are displayed.

758 Figure 6. Distinct differences in the frequency of trials with ESC were demonstrated in the 759 interaction between orientation and location. Standard error bars are displayed.

760 Figure 7. Regardless of location, significantly more ESC was displayed when the handle was 761 oriented towards or to the right compared to away from and to the left of the participant.

762 Standard error bars are displayed. 